\title{
Some experimental results for the end-linked polydimethylsiloxane network system
}

\author{
Anne Ladegaard Larsen ${ }^{1}$ *, Peter Sommer-Larsen ${ }^{2}$, Ole Hassager ${ }^{1}$ \\ ${ }^{1}$ The Technical University of Denmark, Department of Chemical Engineering, Ved \\ Produktionstorvet, bygn. 423, 2800 Lyngby, Denmark; Fax (+45) -4588 2161; \\ all@kt.dtu.dk, oh@kt.dtu.dk \\ ${ }^{2}$ Risø National Laboratory, The Danish Polymer Center, P.O. Box 49, 4000 Roskilde, \\ Denmark; Fax (+45) -4677 4791; peter.sommer.larsen@risoe.dk
}

(Received: May 26, 2004; published: August 11, 2004)

\begin{abstract}
Small-strain equilibrium modulus data of polydimethylsiloxane (PDMS) networks are reviewed. 58 different articles in the literature with values of the modulus of end-linked PDMS networks were found [1-58] including more than 250 values of the small-strain equilibrium modulus as function of variables in the crosslinking system. The dominating variable is shown to be the molecular weight of PDMS as expected, whereas the functionality of the crosslinker methylhydrosiloxane/dimethylsiloxane copolymer is shown to be less important in good agreement with the phantom model. The data collected was fitted to the phenomenological model of Langley and Graessley including an entanglement contribution. The fitted entanglement plateau $(0.16 \mathrm{MPa})$ is in good agreement with literature values and with the theoretical prediction of the tube model. Experimental data for the Mooney-Rivlin constants was found and it was shown that $C_{2}$ is constant with increasing functionality. Furthermore it was found that trapped entanglements can be split into two categories, viz. locked entanglements contributing to the $C_{1}$ term and traditionally trapped entanglements contributing to $C_{2}$.
\end{abstract}

\section{Introduction}

In this work a comparative study of the elastic properties of polydimethylsiloxane (PDMS) networks is made. The networks are formed under different conditions by reaction of difunctional PDMS with the $f$-functional crosslinker methylhydrosiloxane/ dimethylsiloxane copolymer (HMS). In contrast to earlier investigations, many network parameters are evaluated and by applying a few assumptions a comparison of the networks prepared under different conditions is made feasible. The parameters include, e.g., the molecular weight and the molecular weight distribution of the two components, the temperature at which the crosslinking reaction occurred, the use of solvent, the functionality of the crosslinker and the ratio of crosslinker to PDMS. In the following, the different reaction parameters and their influence on the elastic properties are summarized.

The great majority of studies on the mechanical properties of elastomers have been carried out in elongation because of the simplicity of this type of deformation [59], and the most common way to investigate the elastic properties of the network is a 
combination of mechanical stress-strain measurements in elongation and nonmechanical swelling experiments [59-63]. Other possibilities to measure the mechanical properties include dynamic mechanical measurements [1-10] and stressstrain measurements in compression [11-13]. Agreement between theory and experiment has generally been satisfactory [64], but for many elastomers straininduced crystallization occurs, thereby requiring special treatment. Crystallization does not occur in PDMS networks. This - together with other favourable properties such as no side-reactions at low temperature and the possibility of stopping the reaction during the crosslinking reaction - makes PDMS a so-called 'model' network [14,15,65-71].

In addition to mechanical methods, experimental techniques for characterizing polymer networks include IR spectroscopy, small-angle neutron (SANS) [72-80], X-ray (SAXS) $[3,77-84]$ and light scattering studies $[85,86]$. Brillouin scattering $[76,87-$ 90] studies together with investigations of the hyper-sonic viscoelastic behaviour by multi-pass Fabry-Perot spectroscopy (MPFPS) [91] and dielectric relaxation [92] have been performed as well in order to investigate the cross-link density. Birefringence is another relevant optical property of the deformed polymer network [16,93-95]. The strain-induced birefringence can be used to characterize segmental orientation $[94,95]$, both Gaussian and non-Gaussian elasticity, crystallization and other types of chain ordering, and short-range correlations. Spin echo measurements [96] have also been performed on PDMS elastomers, and finally NMR techniques, such as dipolar and quadrupolar interaction ${ }^{2} \mathrm{H}$ NMR [97-101], solid state NMR [84], NMR imaging $[102,103]$ and transverse proton relaxation ${ }^{1} \mathrm{H}$ NMR $[17,103-105]$ have been applied to PDMS networks.

Usually, tetrakis(dimethylsiloxy)silane and phenyltris(dimethylsiloxy)silane are used as 'true' tri- and tetra-functional crosslinkers, respectively, whereas for higher functionalities linear methylhydrosiloxane/dimethylsiloxane copolymer (HMS) chains with different numbers of reactive hydride groups distributed more or less randomly along the chain are used. Short HMS molecules can be regarded as f-functional crosslinkers, whereas long HMS chains with low methylhydrosiloxane content behave as 3-functional, with elastically active chains between the hydride groups, see Fig. 1. Falender et al. [18] and Miller and Macosko [19] investigated HMS crosslinkers with different silane distributions along the chain. They found that the highest modulus was obtained when the silane groups were segregated at the two ends rather than randomly distributed along the chain. This observation agrees with the increased functionality when the groups are segregated in the ends.
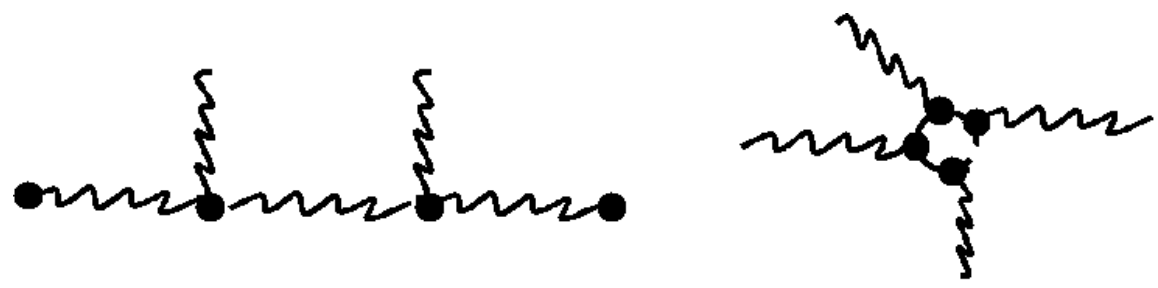

Fig. 1. Low and high density of hydride groups along the HMS chain, respectively. The HMS chains with low density of hydride groups need to be regarded as trifunctional whereas the high density ones can be regarded as $f$-functional

A major problem in the study of most model networks arises from side-reactions [66]. It seems that as much as $30 \%$ of the silane groups may be consumed by various 
side-reactions when the crosslinking reaction was carried out at moderately high temperatures of $65-110^{\circ} \mathrm{C}$ [67-69]. However, no significant side reactions are observed at low temperatures such as $30-35^{\circ} \mathrm{C}$ [66,69-71]. Macosko and Sam [67] observed two major side reactions for the network systems with tetrakis(dimethylsiloxy)silane and phenyltris(dimethylsiloxy)silane, respectively, as crosslinkers, namely a consumption of $\mathrm{Si}-\mathrm{H}$ groups giving redistributed siloxane groups in the resulting polymer, as well as gaseous silanes and siloxanes as by-products, and vinyl-group rearrangement to an internal position within the chain resulting in a loss of reactivity of some vinyl groups. These side-reactions lead to an optimal value, with respect to the highest elastic modulus, of the stoichiometric imbalance of $r_{\mathrm{opt}} \approx 1.3$. The stoichiometric imbalance is the amount of hydride groups relative to the amount of vinyl groups in the reaction mixture. Larsen et al. [20] investigated an 8-functional HMS crosslinker $(2000 \mathrm{~g} / \mathrm{mol})$. They found that no significant side reactions occurred at relatively high temperatures $\left(40 \mathrm{~min}\right.$ at $70^{\circ} \mathrm{C}$ followed by $30 \mathrm{~min}$ at $150^{\circ} \mathrm{C}$ ) and concluded that the reactivity of the linear HMS was lower than that of the usual 'true' crosslinkers. Several authors have reported an optimal value of the stoichiometric imbalance larger than unity despite low reaction temperatures. It seems that a value of $r=1.2-1.4[1-3,20,21,99,106]$ results in the highest modulus rather than the stoichiometric mixture.

Even in computer simulations of end-linked tetra-functional systems, there are conflicting results. The results of Eichinger et al. [107-110] indicate that the most effective elastic networks are produced by stoichiometric systems, whereas simulations of Gilra et al. $[72,113,114]$ indicate that the most effective network is obtained with an excess of crosslinker due to a maximum of ring-formation at $r=1$. However, all studies show that crosslinker-deficient mixtures produce less effective elastomers than those using excess crosslinker. Experiments confirm this observation $[20,69]$.

With the aim of minimizing side reactions, the reaction mixture is usually kept at room temperature for 2 - 3 days or even longer in vacuum. In some studies, the PDMS networks are cured for $2-3$ days at $75^{\circ} \mathrm{C}[10,22,23]$. Furthermore a few authors crosslink the networks in solution [8,9,15,24-27]. If the network is crosslinked in bulk, entanglements are trapped whenever the PDMS chain length exceeds the entanglement molecular weight. On the other hand, in the system where PDMS is crosslinked in solution, the number of trapped entanglements is governed by the polymer concentration [24].

Vasiliev et al. [25] investigated the networks formed in bulk and in solution, respectively, and concluded that the modulus of networks formed in solution is lower than that of a network formed in the bulk state. This was in contrast to the earlier results of Cohen et al. [8] who prepared PDMS networks in the bulk state and with 5 different volume fractions of the polymer mixture as low as $30 \%$ in cyclohexane. They obtained similar results for all networks and concluded that the number of chain entanglements was more or less independent on the degree of dilution. In contrast, later work clearly shows that the modulus does depend upon the degree of dilution. [9,15,24-27]. Vasiliev et al. [25] noticed that the use of a mixture of a good solvent and a non-solvent results in phase separation during crosslinking. The reaction mixture becomes turbid and a porous gel structure remains even after the solvent is removed. Furthermore the prepared networks may be washed by adding a good solvent, e.g., heptane to the network, thereby swelling the network and allowing the unreacted species to diffuse out of the network structure. After the swelling the sample is dried in vacuum. The washing procedure has a large influence on the resulting elastic properties since the removal of inelastic material results in an 
increased density of elastic material in the network sample. This effect is largest for the imperfect networks [115].

Schimmel and Heinrich $[116,117]$ studied the influence of the molecular weight distribution of PDMS chains on the mechanical properties. They concluded that the elastic modulus was lowered with increasing polydispersity. Meyers et al. [28] made a thorough investigation of commercial versus narrow-molecular weight distribution PDMS networks with high functionalities and obtained similar moduli for the two types of network. The same observations were made by Sharaf et al. [118]. It is important to emphasize that the results mentioned above hold for uni-modal molecular weight distributions and are in contrast to the results for bi- and tri-modal networks. Here particularly high extensibilities can be obtained when combining very short chains (molecular weight of a few hundred) and relatively long chains (around 18000) $[29,30,119]$. Apparently, the very short chains are important because of their limited extensibility, and the relatively long chains because of their ability to retard the rupture process $[30,118,119]$. Several other authors have investigated bimodal [14,31-33,120,121] and trimodal [122] PDMS networks as well.

Another well-studied phenomenon [4-6,17,34] is the occurrence of dangling PDMS chains due to either lack of HMS reaction sites or reaction of stoichiometric amounts of mono- and difunctional DMS chains with HMS giving well-defined amounts of dangling material. Diffusion studies of linear PDMS chains in PDMS networks have been performed $[21,35]$ to clarify the diffusion of unattached PDMS chains and thereby obtain an estimate for the characteristic time scales for the PDMS chains in the restricted network.

Studies on systems randomly crosslinked along the PDMS chains in contrast to the end-linked systems have been performed as well [36-38]. No significant differences between randomly crosslinked systems and end-linked systems of the same crosslinking density were observed. The use of a branched crosslinker rather than a linear crosslinker of the same functionality had little effect on the elastic modulus either [28]. Takeuchi and Cohen [39] investigated the difference between hydroxy and vinyl endlinked PDMS, and they found similar swelling properties and moduli.

Llorente and Mark [23] investigated the effect of the crosslinker's functionality on the Mooney-Rivlin constants. For a PDMS chain with $M_{\mathrm{n}}=11300$ and series of crosslinkers with functionality from 3 to 37 they found that $C_{1}$ increased with increasing functionality whereas $C_{2}$ decreased with increasing functionality. These findings are in accordance with the interpretation that the phantom model corresponds to the high-strain limit and the affine model corresponds to the low-strain limit (see Theory section). Meyers et al. [28] did not observe any significant change in $C_{2}$ with the functionality for PDMS chains with $M_{\mathrm{n}}=21600$ suggesting an entanglement contribution to $C_{2}$ as well as $C_{1}$. They observed the same tendency as Llorente and Mark for chains with $M_{\mathrm{n}}=11100$, where the number of entanglements is low. Thorough studies on the gelation process of PDMS networks have been performed as well [69,70,123-126].

In literature several articles summarizing results for PDMS networks have been found $[2,106,117,118,127-130]$ but none of the articles is very extensive, focusing on the effect of molecular weight or functionality only upon the elastic modulus. To facilitate comparison of the experimental data as a function of both $f$ and $M$, a few assumptions are needed:

1. All crosslinker molecules are expected to behave the same except for their different functionalities, i.e., the distribution and the density of reaction sites along the 
chains are assumed not to influence the resulting networks. The molecular weight of the crosslinkers is furthermore assumed to be vanishing in comparison with the molecular weight of PDMS, so that the molar concentration of possible elastic chains is inversely related to the molecular weight of PDMS.

2. The data is assumed to come from the strongest network obtainable, not necessarily the network composed from a stoichiometric reaction mixture. This assumption is necessary since some authors do not state the stoichiometry due to the lack of precise molecular weight determinations and hence find the strongest network by making networks with different ratios of HMS to DMS, while other authors determine the molecular weight and use a stoichiometric mixture and not the stoichiometry for obtaining the strongest network.

3. The reaction conditions (temperature, time, solvent or not, catalyst etc.) do not influence the properties of the final network.

4. Polydispersity is not taken into account, only the number-average molecular weight of PDMS. However, networks resulting from bimodal distributed PDMS are not included since they behave significantly different from polydisperse PDMS networks.

5. Washed and non-washed elastomers have the same properties. If coherent data from both washed and non-washed networks are available, the data from nonwashed networks are used since the data of non-washed networks are far more common.

As it can be seen in two recent articles [20,39], assumption 3 can easily cause a factor 2 in deviation between data obtained from reaction mixtures with $r=1$ and $r=$ $r_{\mathrm{opt}}$, respectively, whereas the other assumptions should have a reduced effect on the modulus.

\section{Theory}

The traditional theories of rubber elasticity have been developed on the basis of network chains being simple Gaussian random coils. Models of elastomers in the 'phantom' limit (in which the chains are assumed to be devoid of material properties) were developed by James and Guth [131]. In such a phantom network, it is assumed that the chains move freely through one another, and that the only contribution to the elasticity arises from the network connectivity. The phantom model predicts the modulus to be proportional to the inverse of the molecular weight of the prepolymer:

$$
G_{\mathrm{ph}}=(v-\mu) R T=\left(1-\frac{2}{f}\right) \frac{\rho}{M} R T
$$

where $\mu$ is the amount of junctions per unit volume given by $2 /(f \cdot v)$ with $f$ being the functionality, $v$ the number of elastic chains per unit volume, $R$ the gas constant, $T$ the absolute temperature, $\rho$ the density of PDMS, and $M$ the molecular weight.

In an affine network, on the other hand, the end-to-end chain vectors are assumed to transform affinely with the macroscopic deformation. The affine model does not take into account the functionality of the crosslinking agent:

$$
G_{\mathrm{af}}=v R T=\frac{\rho}{M} R T
$$

It can be seen that for high functionalities, the affine and the phantom model coincide. The phantom and affine limits of deformation are two extreme cases, and 
experimental stress-strain measurements suggest that low-molecular-weight PDMS networks exhibit properties between these two limits [106]. The network is predicted to be more affine-like at small deformations and more phantom-like at larger deformations. Contributions to the modulus from points along the contour of the polymer chains are not considered in the two above models, and these so-called entanglements turn out to contribute significantly to the elastic modulus when the molecular weight of the PDMS prepolymer is increased. Langley [132] and Langley and Graessley [133] developed a phenomenological model with an additional term introduced to allow for the contribution from entanglements. The result of the model can be expressed as:

$$
G_{\mathrm{LG}}=(v-h \mu) R T+G_{\mathrm{o}} T_{\mathrm{E}}
$$

where $h$ is an empirical parameter between 0 and $1, G_{0}$ is the plateau modulus of the melt of uncrosslinked, high-molecular-weight prepolymer, and $T_{\mathrm{E}}$ is the proportion of the maximum concentration of topological interactions that contribute to the modulus, the so-called trapping factor. This result indicates that with increased molecular weight of PDMS, a plateau is obtained:

$$
G_{M \rightarrow \infty}=G_{0} T_{E}
$$

The entanglement molecular weight $M_{\mathrm{E}}$ is determined from the equilibrium modulus of long, linear chains $G_{0}$ as:

$$
G_{0}=\frac{\rho}{M_{E}} R T
$$

Several values for the entanglement molecular weight have been found in literature ranging from 10100 to 12500 [134-137] and we use in the following a value of 12000.

Stress-strain data is usually interpreted in terms of the reduced stress defined as the ratio of the true stress difference $\left(\sigma_{\mathrm{zz}}-\sigma_{\mathrm{rr}}\right)$ to the corresponding difference of the Finger strain tensors $\left(\beta_{\mathrm{zz}}-\beta_{\mathrm{rr}}\right)$ :

$$
f^{*}=\frac{\sigma_{\mathrm{zz}}-\sigma_{\mathrm{rr}}}{\beta_{\mathrm{zz}}-\beta_{\mathrm{rr}}}=\frac{F}{A_{\mathrm{o}}\left(\alpha-\alpha^{-2}\right)}
$$

where $F$ is the measured force, $A_{\circ}$ is the initial cross-sectional area of the unstretched sample, and $\alpha$ is the relative length of the sample.

For a neo-Hookean material, the reduced stress is independent of the stretch ratio. More generally, the reduced stress is interpreted in terms of the semi-empirical Mooney-Rivlin (MR) equation [138,139]:

$$
f^{*}=2 C_{1}+2 C_{2} \alpha^{-1}
$$

where $C_{1}$ and $C_{2}$ are constants independent of $\alpha$. To get precise determinations of the $C_{2}$ constant, relatively long extensions are required.

Introducing an additional factor describing the network results in new expressions for the MR constants defined by the trapping factor and the new locking factor. The locking factor $L_{E}$ is defined as the fraction of trapped entanglements being locked. The fraction of trapped entanglements that are locked contributes to the $2 C_{1}$ term, while the other fraction not being trapped contributes to the $2 C_{2}$ term due to their capability of sliding. The Mooney-Rivlin constants can then be written as: 
$2 C_{1}=\left(1-\frac{2}{f}\right) V R T+G_{\mathrm{o}} T_{\mathrm{E}} L_{\mathrm{E}}$

$2 C_{2}=\left(1-L_{E}\right) G_{0} T_{E}$

Another approach to describe the elasticity of polymers in general is the tube model. The tube model predicts the modulus to be [144-146]:

$$
G=v R T\left(\frac{4}{3} \cdot \frac{2 N+1}{3 N+1}+\frac{1}{5}(N-1) \frac{11 N+5}{3 N+1}\right)
$$

where $N=M I M_{\mathrm{E}}$ is the number of entanglements per chain. Introducing the expression for the crosslink concentration leads to:

$$
\frac{G M_{E}}{\rho R T}=\frac{G}{G_{0}}=\frac{1}{N}\left(\frac{4}{3} \cdot \frac{2 N+1}{3 N+1}+\frac{1}{5}(N-1) \frac{11 N+5}{3 N+1}\right)
$$

The tube model predicts a modulus in the entangled regime $(N>>1)$ of $(11 / 15) \cdot G_{0}$ and thereby a trapping factor of $11 / 15=0.73$. The prefactor or trapping factor is nearly identical to the result obtained by the slip-link model of Higgs and Ball [147] and Edwards and Vilgis [148] although they started from a completely different set of physical assumptions and mathematical framework.

Eq. (3) covers a lot of models. The adjustable parameter $h$ makes it possible to alter the properties of the network gradual from affine $(h=0)$ to phantom $(h=1)$ behaviour. The trapping factor in the entanglement contribution can be interpreted in terms of statistics (as proposed by Langley et al.) or be derived from the slip-link or the tube model.

Swelling equilibria are usually interpreted by means of an equation $[60,61]$ that balances osmotic pressure and elastic stresses:

$$
M_{\mathrm{n}}=\frac{C \rho V_{1}\left(v_{2 m}^{1 / 3}-\omega v_{2 m}\right)}{\ln \left(1-\omega v_{2 m}\right)+v_{2 m}+X v_{2 m}^{2}}
$$

where $V_{1}$ is the molecular volume of the swelling agent. $X$ is the Flory-Huggins interaction parameter, and the parameter $\omega$ takes into account a possible volumedependent contribution to the free energy of the network. Usually this parameter is omitted, and experimental data on PDMS networks from Mark and Sullivan [41] indicates that this is reasonable. $v_{2 m}$ is the volume fraction of polymer at swelling equilibrium. $C$ is the structural factor, i.e., $C=C_{1}$ if the affine model is applied, and $C$ $=1-2 / f$ if the phantom model is used. The factor can be determined from experimental results if the $C_{1}$ constant of the Mooney-Rivlin equation is determined from stress-strain data:

$$
2 \mathrm{C}_{1}=C \rho R T M_{\mathrm{n}}^{-1}
$$

The above relations are used to convert swelling data into traditional modulus data.

\section{Results and discussion}

We compare experimental results for networks with functionalities ranging from 3 to 83.6 with the predictions of the phantom (Eq. (1)), the affine (Eq. (2)), and the Langley-Graessley (Eq. (3)) models. All data is measured at room temperature or 
converted to room temperature by traditional time-temperature superposition. The data is extracted from a total of 57 papers. A summary of the data collected can be seen in Tab. 1.

Tab. 1. The experimental data collected. $x_{\text {below }}$ denotes the fraction of data points below the least-square fit of all the data to the Langley-Graessley model

\begin{tabular}{cccc}
\hline Functionality & $\begin{array}{c}\text { Number of } \\
G \text { data }\end{array}$ & $\begin{array}{c}\text { Number of } \\
\text { MR data }\end{array}$ & $x_{\text {below }}$ \\
\hline 3 & 26 & 4 & 0.85 \\
$3-4$ & 13 & 10 & 0.77 \\
4 & 116 & 10 & 0.63 \\
5 & 9 & - & 0.12 \\
$6-10$ & 8 & 6 & 0.15 \\
$11-20$ & 7 & 3 & 0.17 \\
$21-25$ & 31 & 24 & 0.08 \\
$26-39$ & - & - & - \\
$>40$ & 61 & 35 & 0.07 \\
\hline total & 264 & 92 & 0.47
\end{tabular}

Since investigations are most extensive for 3- and 4-functional networks, we treat them separately. In Figs. 2 and 3, the experimental data for 3- and 4-functional networks are fitted to the Langley-Graessley form with $h=1$, i.e., $G(M)=a(1-2 / f) /(M+$ b). The least-square fits are given by:

$$
\begin{aligned}
& G_{f=3} /[\mathrm{MPa}]=\frac{1}{3} \frac{(2.55 \pm 0.02) \cdot 10^{3}}{M_{\text {DMS }}}+(0.069 \pm 0.002) \\
& G_{f=4} /[\mathrm{MPa}]=\frac{1}{2} \frac{(2.38 \pm 0.04) \cdot 10^{3}}{M_{\text {DMS }}}+(0.122 \pm 0.005)
\end{aligned}
$$

There is good agreement in the fitted a parameters whereas the plateau moduli $(b)$ differ significantly. This can be explained by either an $f$-dependency of the plateau modulus or by the fact that the average molecular weight of the data from the 3functional network is 13500 whereas for the 4-functional network it is 18277 . The average molecular weight of the 3-functional data is only slightly above the entanglement molecular weight, so entanglements may not interact as much as for the 4functional networks where the average molecular weight is well above the entanglement molecular weight.

All the data collected is fitted to an equation of the Langley-Graessley form with $h=$ 1 , and the fit and experimental data can be seen in Fig. 4. The average molecular weights of the data range from 660 to 84000 with an average value of 18400 . The expression obtained is:

$$
G /[\mathrm{MPa}]=\left(1-\frac{2}{f}\right) \frac{(2.36 \pm 0.06) \cdot 10^{3}}{M_{\mathrm{DMS}}}+(0.142 \pm 0.007)
$$




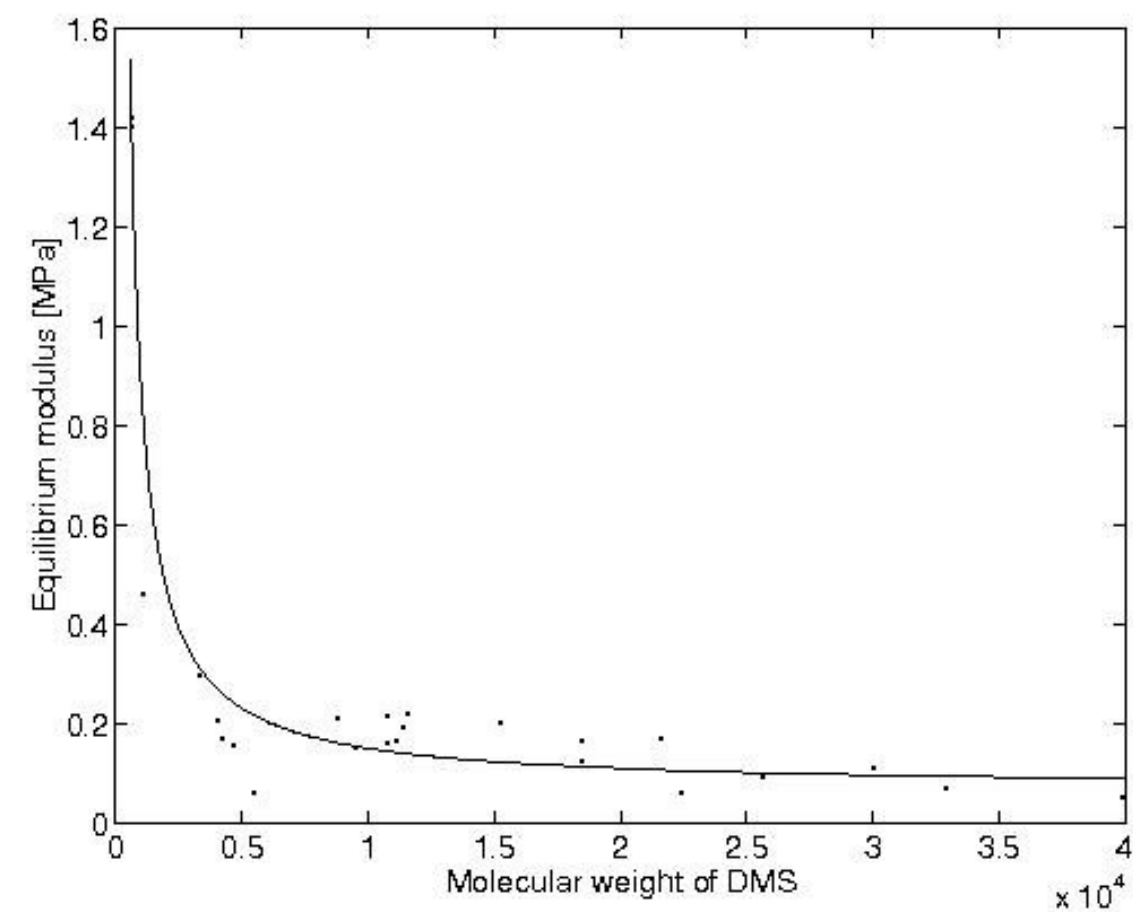

Fig. 2. Experimental data for the 3-functional crosslinker for $r_{\text {opt }}$ compared to a fit obtained from a least-square minimization of the function: $G=a(1-2 / f) /\left(M_{\mathrm{DMS}}+b\right) .24$ data points were collected with an average value of the molecular weight of 13500

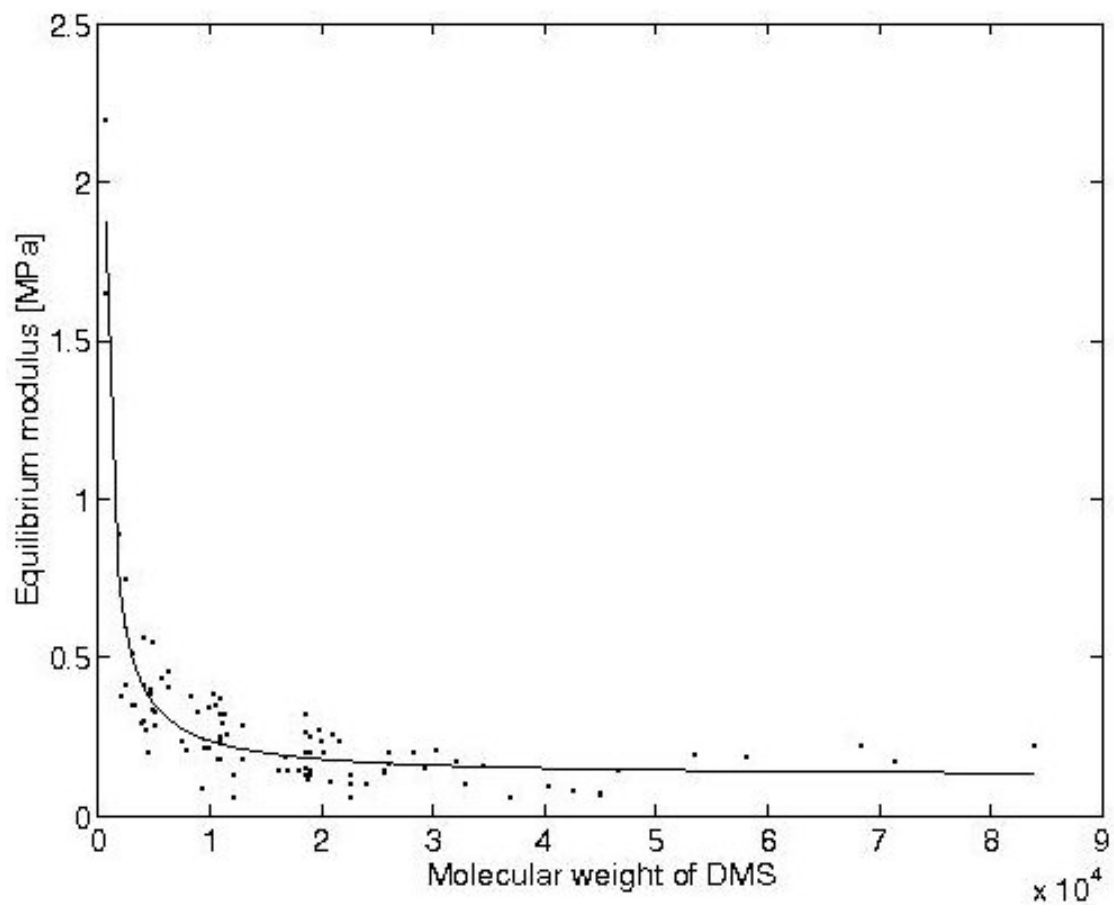

Fig. 3. Experimental data for the 4-functional crosslinker for $r_{\mathrm{opt}}$ compared to a fit obtained from a least-square minimization of the function: $G=a(1-2 / f) /\left(M_{D M S}+b\right) .86$ data points were collected with an average value of the molecular weight of 18200

Compared to the phantom model combined with the plateau modulus of a wellentangled PDMS melt (entanglement molecular weight 12000 and plateau modulus 
at $298 \mathrm{~K}$ equal to $0.200 \mathrm{MPa}$ ) it can be seen that the agreement is striking if the trapping factor of 0.73 as predicted by the tube model is used:

$G_{\text {theo }} /[\mathrm{MPa}]=\left(1-\frac{2}{f}\right) \frac{2428}{M_{\text {DMS }}}+0.147$

It is clear from Eqs. (12), (13), and (14) that the factor a seems to be decreasing with functionality whereas the factor $b$ seems to increase with functionality. This indicates that either there may be more terms in the expression or that the $h$ parameter needs to be fitted as well. It turns out that optimizing on $a, b$, and $h$ gives a value of $h=1.05$ very close to the assumption of $h=1$ applied previously. Adding two terms of the form $b / M$ and $c / f$ and minimizing gives the results in Tab. 2. From this it is clear that no significant improvement of the fit is obtained by the inclusion of additional parameters, but that the decrease is caused by statistical uncertainties within the applied data since the average molecular weights are not the same for the three data sets ( $f$ $=3, f=4$ and all data). The value of $0.147 \mathrm{MPa}$ is in good agreement with the results of Venkatraman [140], who presented an empirical equation relating the volume fraction of polymeric network $\Phi_{\text {net }}$ to the trapped entanglement contribution to the modulus: $G_{\mathrm{TE}}\left(\Phi_{\text {net }}\right) /[\mathrm{MPa}]=0.12 \Phi_{\text {net }}^{2.4}$ resulting in a plateau of $0.12 \mathrm{MPa}$ given a perfect network $\left(\Phi_{\text {net }}=1\right)$, and with Mark et al. [76] who performed a Brillouin scattering study of PDMS and determined the modulus of long-range interactions and entanglements to be of the order $0.1 \mathrm{MPa}$.

Tab. 2. Least square minimization fits of the experimental data to equations of the form: $G=(1-2 / f) a / M+b / M+c / f+d .^{\diamond}$ and ${ }^{\circ}$ are fits to the affine and phantom models, respectively. ${ }^{*}$ denotes that $h$ is fitted as well

\begin{tabular}{ccccc}
\hline$a$ & $b$ & $c$ & $d$ & $\sum$ (residuals) $^{2}$ \\
\hline$\diamond-$ & 1299 & - & - & 8.40 \\
$\circ 3131$ & - & - & - & 4.55 \\
- & 952 & - & 0.180 & 3.00 \\
2343 & - & - & 0.142 & 1.76 \\
$* 2405$ & - & - & 0.145 & 1.71 \\
3340 & -443 & - & 0.132 & 1.61 \\
2368 & - & -0.365 & 0.211 & 1.41 \\
2354 & 5.36 & -0.367 & 0.212 & 1.41
\end{tabular}

Tab. 3. Least square minimization fits of the experimental data to equations of the form: $2 C_{1}=(1-2 / f) a / M+b / M+c / f+d$

\begin{tabular}{ccccc}
\hline$a$ & $b$ & $c$ & $d$ & $\sum$ (residuals) $^{2}$ \\
\hline 2899 & - & 0.0555 & - & 0.71 \\
2476 & - & - & 0.0785 & 0.34 \\
2448 & - & -0.326 & 0.114 & 0.22 \\
2348 & 0.0496 & -0.0003 & 0.0001 & 0.24 \\
4565 & -1004 & - & 0.0433 & 0.089
\end{tabular}


Tab. 4. Least square minimization fits of the experimental data to equations of the form: $2 C_{2}=(1-2 / f) a / M+b / M+c / f+d$

\begin{tabular}{ccccc}
\hline$a$ & $b$ & $c$ & $d$ & $\sum$ (residuals) $^{2}$ \\
\hline- & - & - & 0.090 & 0.068 \\
0.0140 & - & - & 0.089 & 0.068 \\
-0.0138 & - & 28.07 & 0.088 & 0.066 \\
186.7 & -56.0 & - & 0.083 & 0.064 \\
272.7 & -97.9 & 0.0206 & 0.080 & 0.064
\end{tabular}

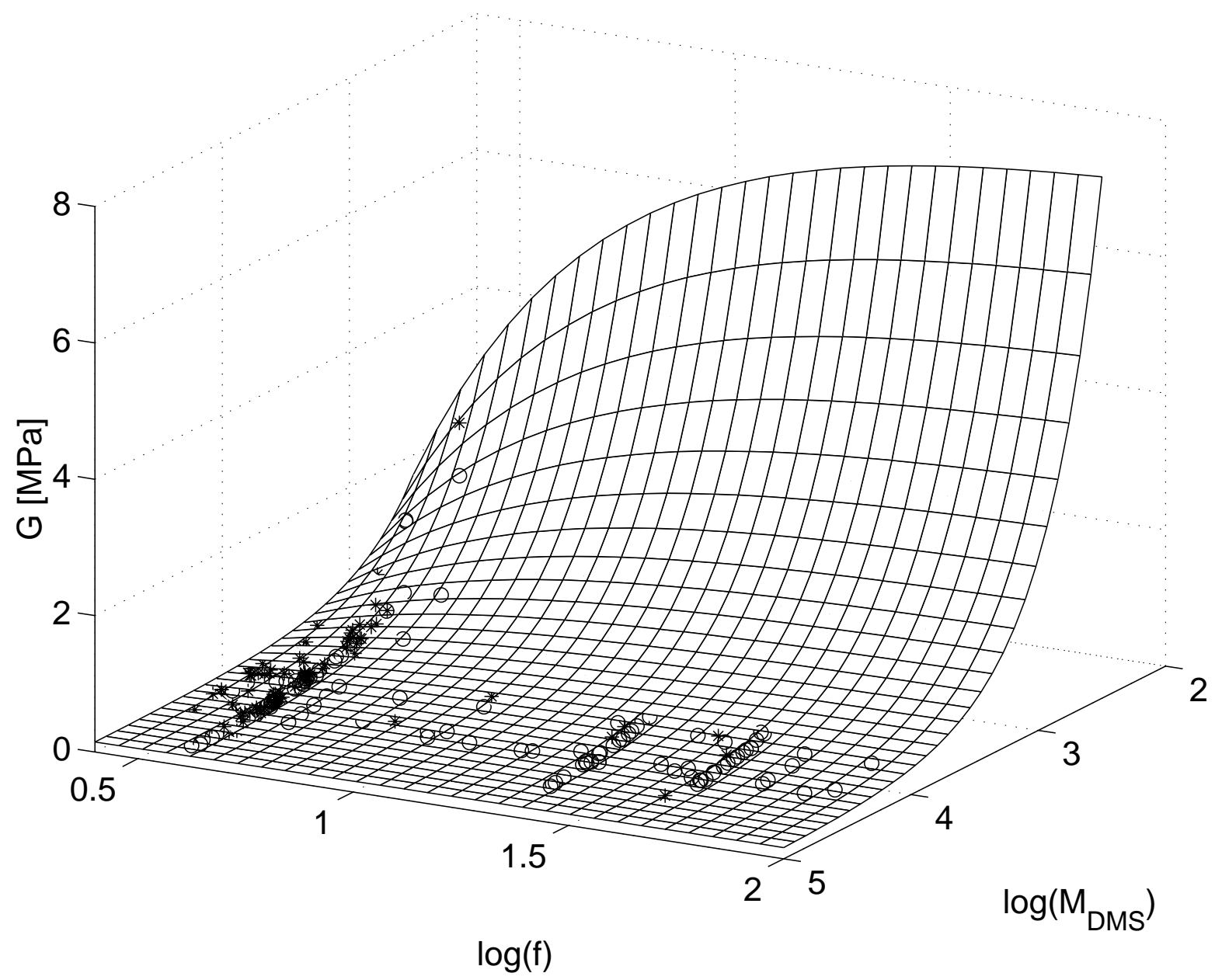

Fig. 4. Experimental data compared to a fit obtained from the least-square minimization of the function: $G=a / M_{\mathrm{DMS}}+b$. Circles and stars indicate that the experimental results are below and above the fit, respectively. 222 data points were collected

From Fig. 5 , it is observed that for $(1-2 / f) / M<1.9 \cdot 10^{-4}$ the network is dominated by trapped entanglements. For a four-functional network, this means that $M>26300$ results in a network dominated by trapped sliding links. That is approximately two entanglement lengths. The network obtained in this domain will be soft and able to extend to a large extent due to the sliding temporary crosslinks. From Tab. 3 , it is clear that there is no additional $f$-dependency of $2 C_{1}$. Furthermore different powers of 
$f$ have been investigated but still there is no indication of improvement of the fits. From this it can be concluded that $2 C_{1}$ can be described as:

$$
\begin{aligned}
& 2 C_{1} /[\mathrm{MPa}]=\left(1-\frac{2}{f}\right) \frac{(2.48 \pm 0.05) \cdot 10^{3}}{M_{\mathrm{DMS}}}+(0.079 \pm 0.007) \\
& 2 C_{2} /[\mathrm{MPa}]=\left(1-\frac{2}{f}\right) \frac{(7 \pm 2) \cdot 10}{M_{\mathrm{DMS}}}+(0.085 \pm 0.003) \cong 0.085 \pm 0.003
\end{aligned}
$$

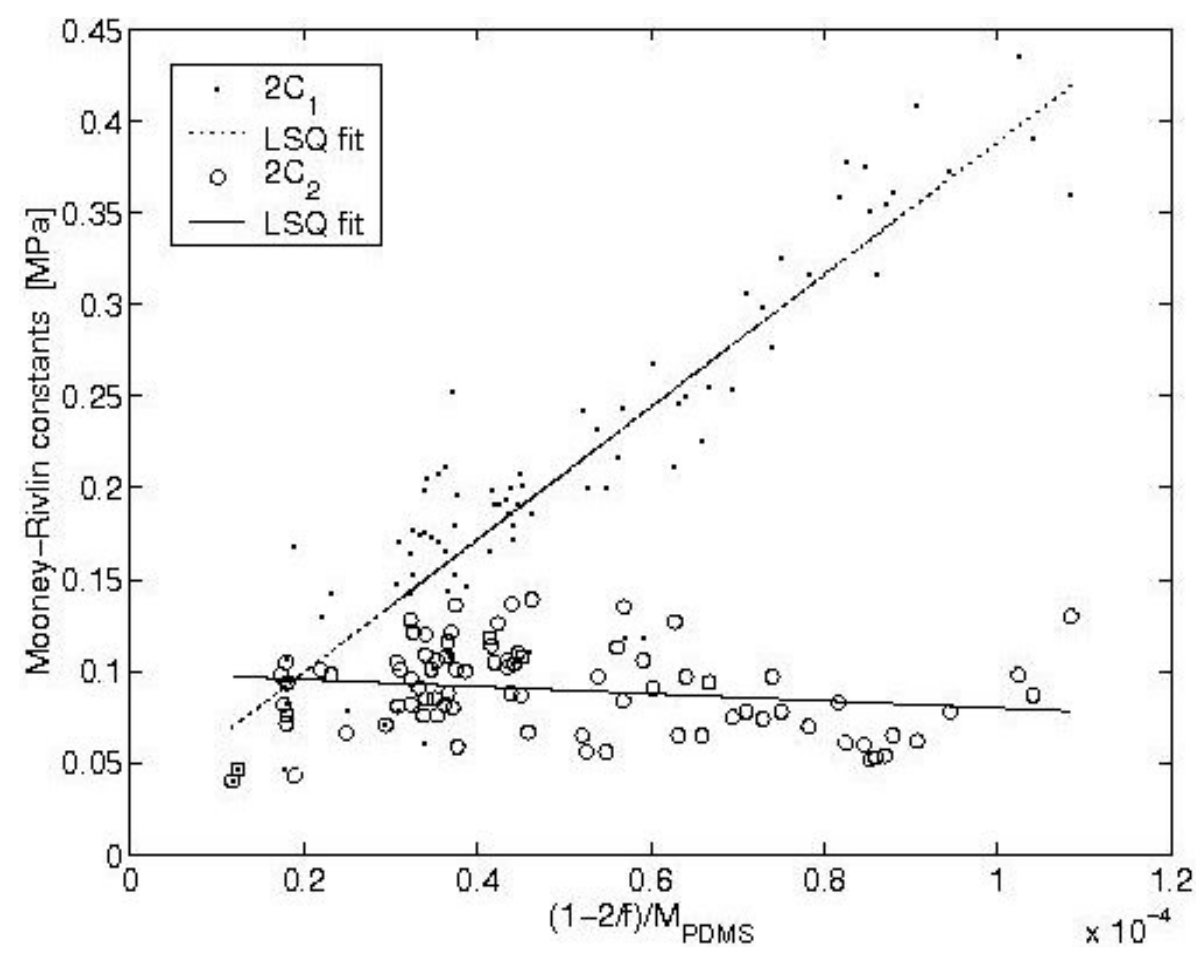

Fig. 5. Experimental data for the MR constants compared to a fit obtained from the least-square minimization. The intercept of the two fits indicates the transition between domination of trapped entanglements and crosslinks

The data and fits can be seen in Fig. 6 together with a fit of the affine model to the $2 C_{1}$ constant. It can be seen that the plateau modulus predicted from $2 C_{1}+2 C_{2}$ is in good agreement with the plateau modulus predicted taking into account all experimental data, and furthermore the plateau modulus of $2 C_{1}$ is very close to the value of 0.12 according to the result of Venkatraman [140]. The agreement between the affine model and the $2 C_{1}$ constant is poor. This observation clearly supports the previous results from Larsen et al. [20] who stated that trapped entanglements fall into two categories: locked entanglements and slip-links contributing to the $2 C_{1}$ and $2 C_{2} \mathrm{MR}$ constants, respectively. Hereby a constant contribution arising from entanglements needs to be added to the contribution from chemical crosslinks. The result that $2 C_{2}$ does not depend significantly on either $f$ or $M_{\mathrm{DMS}}$ is remarkable. The non-dependence on functionality may be seen as evident for the existence of slip-links. Slip-links can be regarded as 4-functional temporary crosslinks and will not depend on the functionality of the HMS chain. The concentration of slip-links (a fraction of the existing entanglements) will be constant with increasing $M_{\mathrm{DMS}}$, which is observed as well. From the least-square fits, the trapping factor is determined to 0.80 and the locking factor to 0.48 . This result indicates that half of the trapped entanglements act similar 
to chemical crosslinks, and that the other half is capable of sliding and therefore release stress during extension.

In Fig. 7, the prediction of the stochastic model from Larsen et al. [20] with the determined $T_{E}$ and $L_{E}$ from the least-square fit can be seen. Obviously, the value of the locking factor is in very good agreement with experimental data not only for perfect networks.

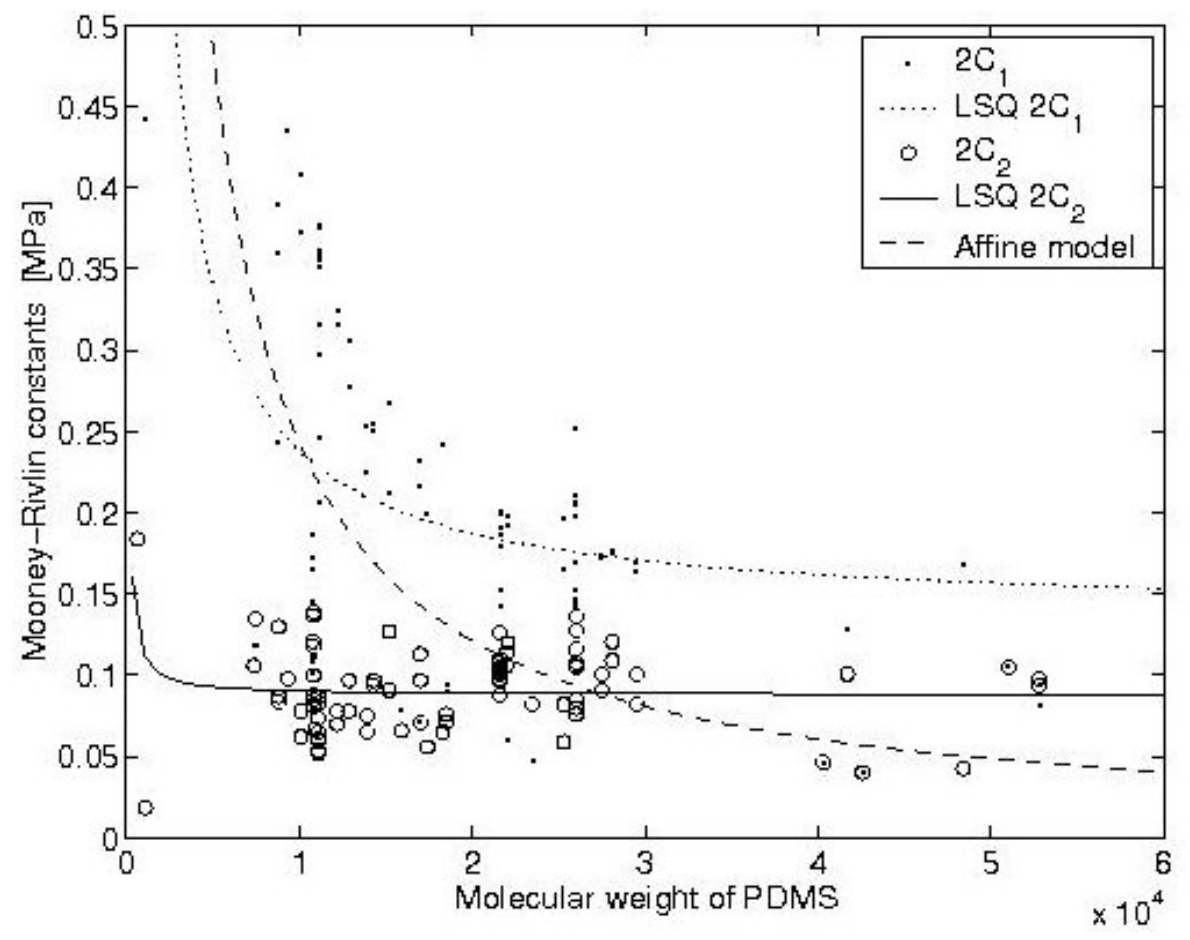

Fig. 6. Experimentally determined Mooney-Rivlin constants compared to the affine network model. LSQ $2 C_{i},(i=1,2)$ is a least square minimization of the collected MR data to the elastic modulus of the Langley-Graessley form. 92 data points were collected
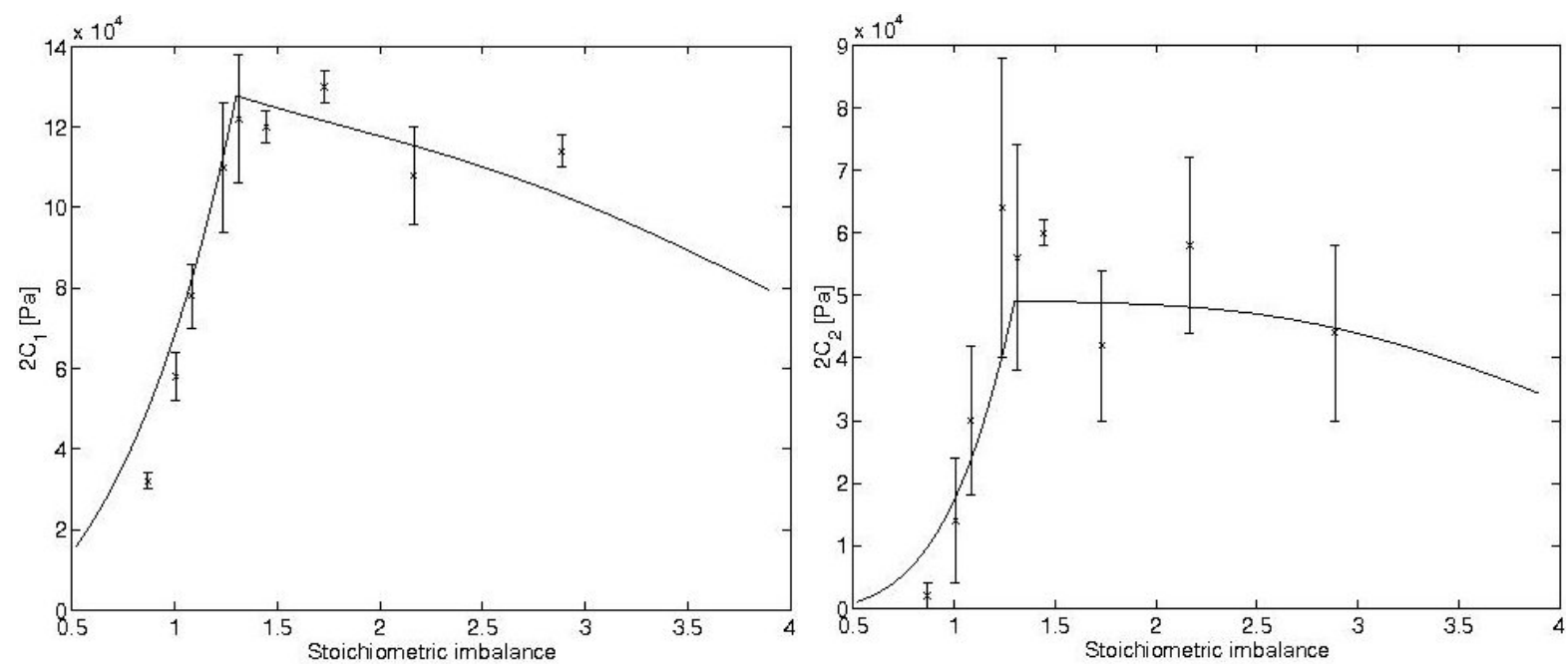

Fig. 7. The Mooney-Rivlin constants as functions of stoichiometric imbalance compared to a stochastic model given a trapping factor of 0.80 and a locking factor of 0.48. The stochastic model is shifted right a factor of $r_{\text {opt. }}$. Data is published in ref. [20] 


\section{Conclusion}

The agreement between the collected data and the Langley-Graessley model is reasonable despite the many variable parameters in the network system. It seems reasonable to compare the modulus data as function of two variables only, namely the functionality of HMS and the molecular weight of PDMS. Clear evidence was found that the elastic moduli of almost perfect PDMS networks have a constant contribution from entanglements and hence can neither be described by the phantom model nor the affine model. Applying the phantom model and adding a contribution from entanglements gives reasonable agreement between experimental data and model. The entanglements contribute with approximately 0.8 of the plateau modulus of a well-entangled melt with the same backbone structure. This observation is in very good agreement with the theoretical prediction from the tube model, which predicts a factor of $11 / 15=0.73$. From the Mooney-Rivlin constants it is clear that it is not chemical crosslinks only that contribute to the $2 C_{1}$ constant but locked entanglements contribute as well. The contribution to the elastic modulus from slip-links is

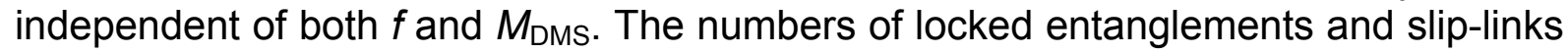
are similar.

[1] Macosko, C. W.; Benjamin, G. S.; Pure Appl. Chem. 1981, 53, 1505.

[2] Gottlieb, M.; Macosko, C. W.; Benjamin, G. S., Meyer, K. O.; Merrill, E. W.; Macromolecules 1981, 14, 1039.

[3] Patel, S. K.; Malone, S.; Cohen, C.; Gillmor, J. R.; Colby, R. H.; Macromolecules 1992, 25, 5241.

[4] Villar, M. A.; Vallés, E. M.; Macromolecules 1996, 29, 4081.

[5] Urayama, K.; Yokoyama, K.; Kohjiya, S.; Macromolecules 2001, 34, 4513.

[6] Granick, S.; Pedersen, S.; Nelb, G. W.; Ferry, J. D.; J. Polym. Sci. B 1981, 19, 1745.

[7] Sullivan, J. L.; Mark, J. E.; Hampton, P. G.; Cohen, R. E.; J. Chem. Phys. B 1978, 68, 2010.

[8] Cohen, R. E.; Severson, S. D.; Yu, C. U.; Mark, J. E.; Macromolecules 1977, 10, 663.

[9] Urayama, K.; Kawamura, T.; Kohjiya, S.; J. Chem. Phys. B 1996, 105, 4833.

[10] Rouf-George, C.; Munch, J. P.; Beinert, G.; Isel, F.; Pouchelon, A.; Palierne, J. F.; Boué, F.; Bastide, J.; Polymer Gels and Networks 1996, 4, 435.

[11] Rennar, N.; Ber. Bunsenges., Phys. Chem. 1998, 102, 1665.

[12] Pak, H.; Flory, P. J.; J. Polym. Sci. B 1979, 17, 1845.

[13] Menge, H.; Hotopf, S.; Pönitzsch, S.; Richter, S.; Arndt, K.-F.; Schneider, H.; Heuert, U.; Polymer 1999, 40, 5303.

[14] Andrady, A. L.; Llorente, M. A.; Mark, J. E.; J. Chem. Phys. B 1980, 72, 2282.

[15] Johnson, R. M.; Mark, J. E.; Macromolecules 1972, 5, 41.

[16] Erman, B.; Mark, J. E.; Macromolecules 1998, 31, 3099.

[17] Vega, D. A.; Villar, M. A.; Vallés, E. M.; Steren, C. A.; Monti, G. A.; Macromolecules 2001, 34, 283.

[18] Falender, J. R.; Yeh, G. S. Y.; Mark, J. E.; J. Am. Chem. Soc. 1979, 101, 7353. 
[19] Miller, D. R.; Macosko, C. W.; J. Polym. Sci. B 1987, 25, 2441.

[20] Larsen, A. L.; Hansen, K.; Sommer-Larsen, P.; Hassager, O.; Bach, A.; Ndoni, S.; Joergensen, M.; to be published.

[21] Gent, A. N.; Tobias, R. H.; J. Polym. Sci. B 1982, 20, 2317.

[22] Kawamura, T.; Urayama, K.; Kohjiya, S.; Macromolecules 2001, 34, 8252.

[23] Llorente, M. A.; Mark, J. E.; Macromolecules 1980, 13, 681.

[24] Urayama, K.; Kohjiya, S.; J. Chem. Phys. B 1996, 104, 3352.

[25] Vasiliev, V. G.; Rogovina, L. Z.; Slonimsky, G. L.; Polymer 1985, 26, 1667.

[26] Sivasailam, K.; Cohen, C.; J. Rheol. 2000, 44, 897.

[27] Mark, J. E.; McCarthy, D. W.; Rubber Chemistry and Technology 1998, 71, 928.

[28] Meyers, K. O.; Bye, M. L.; Merrill, E. W.; Macromolecules 1980, 13, 1045.

[29] Sharaf, M. A.; Mark, J. E.; Al-Ghazal, A. A.-R.; J. Appl. Polym. Sci., Appl. Polym. Symp. 1994, 55, 139.

[30] Besbes, S.; Bokobza, L.; Monnerie, L.; Bahar, I.; Erman, B.; Macromolecules 1995, 28, 231.

[31] Wang, S.; Mark, J. E.; J. Polym. Sci. B 1992, 30, 801.

[32] Llorente, M. A.; Andrady, A. L.; Mark, J. E.; J. Polym. Sci. B 1981, 19, 621.

[33] Andrady, A. L.; Llorente, M. A.; Mark, J. E.; J. Chem. Phys. B 1980, 73, 1439.

[34] Larsen, A. L.; Sommer-Larsen, P.; Hassager, O.; 'H-Polymer model applied to networks', to be published.

[35] Mazan, J.; Leclerc, B.; Galandrin, N.; Courraze, G.; Eur. Polym. J. 1995, 31, 803.

[36] Langley, N. R.; Polmanteer, K. E.; J. Polym. Sci. B 1974, 12, 1023.

[37] Oppermann, W.; Rehage, G.; in "Elastomer and Rubber Elasticity", Mark, J. E.; Lal, J.; editors; ACS Symp. Ser. 1982, 193, 309.

[38] Gottlieb, M.; Macosko, C. W.; Lepsch, T. C.; J. Polym. Sci. 1981, 19, 1603.

[39] Takeuchi, H.; Cohen, C; Macromolecules 1999, 32, 6792.

[40] Hedden, R. C.; Saxena, H.; Cohen, C.; Macromolecules 2000, 33, 8676.

[41] Mark, J. E.; Sullivan, J. L.; J. Chem. Phys. B 1977, 66, 1006.

[42] Hedden, R. C.; Wong, C.; Cohen, C.; Macromolecules 1999, 32, 5154.

[43] Tang, M.-Y.; Mark, J. E.; Macromolecules 1984, 17, 2616.

[44] Tang, M.-Y.; Garrido, L.; Mark, J. E.; Polymer 1984, 25, 347.

[45] Mark, J. E.; Macromolecules 1984, 17, 2924.

[46] Mark, J. E.; Tang, M.-Y.; J. Polym. Sci. B 1984, 22, 1849.

[47] Jiang, C.-Y.; Garrido, L.; Mark, J. E.; J. Polym. Sci. B 1984, 22, 2281.

[48] Mark, J. E.; Kato, M.; Ko, J. H.; J. Polym. Sci. B 1976, 54, 217.

[49] Llorente, M. A.; Andrady, A. L.; Mark, J. E.; Colloid Polym. Sci. 1981, 259, 1056.

[50] Kawamura, T.; Urayama, K.; Kohjiya, S.; J. Polym. Sci. B 2002, 40, 2780.

[51] Vallés, E. M.; Macosko, C. W.; Macromolecules 1979, 12, 673.

[52] Zhang, Z. M.; Mark, J. E.; J. Polym. Sci. B 1982, 20, 473. 
[53] Sharaf, M. A.; Mark, J. E.; Alshamsi, A. S.; Polym. J. 1996, 28, 375.

[54] Nguyen, Q. T.; Bendjama, Z.; Clement, R.; Ping, Z.; Phys. Chem. B 1999, 2761.

[55] Meyers, K. O.; Ph.D. Thesis, Massachusetts Institute of Technology, 1980.

[56] Kirk, K. A.; Bidstrup, S. A.; Merrill, E. W.; Meyers, K. O.; Macromolecules 1982, 15, 1123.

[57] Rennar, N.; Oppermann, W.; Colloid. Polym. Sci. 1992, 270, 527.

[58] Oppermann, W.; Rehage, G.; Preprints, IUPAC Symposium, Mainz, Sept. 1979.

[59] Mark, J. E.; Erman, B.; "Rubberlike Elasticity; A Molecular Primer", Wiley-Interscience, New York 1988.

[60] Flory, P.J.; "Principles of Polymer Chemistry", Cornell University Press, Ithaca, NY 1953.

[61] Treloar, L. R. G.; "The Physics of Rubber Elasticity", $3^{\text {rd }}$ edition, Clarendon Press, Oxford, UK 1975.

[62] Kloczkowski, A.; Sharaf, M. A.; Mark, J. E.; Chem. Eng. Sci. 1994, 49, 2889.

[63] Hild, G.; Polymer 1997, 38, 3279.

[64] Mark, J. E.; J. Phys. Chem. B 2003, 107, 903.

[65] Erman, B.; Mark, J. E.; "Structures and Properties of Rubberlike Networks", Oxford University Press, New York 1988.

[66] Fischer, A.; Gottlieb, M.; Proc. of Networks 1986, Denmark.

[67] Macosko, C. W.; Saam, J. C.; Polym. Bull. (Berlin) 1987, 18, 463.

[68] Aranguren, M. I.; Macosko, C. W.; Macromolecules 1988, 21, 2484.

[69] Venkataraman, S. K.; Coyne, L.; Chambon, F.; Gottlieb, M.; Winter, H. M.; Polymer 1989, 30, 2222.

[70] Winter, H. H.; Chambon, F.; J. Rheol. 1987, 31, 683.

[71] Chambon, F.; Winter, H. H.; J. Rheol. 1986, 30, 367.

[72] Gilra, N.; Cohen, C.; Briber, R. M.; Bauer, B. J.; Hedden, R. C.; Panagiotopoulos, A. Z.; Macromolecules 2001, 34, 7773.

[73] Falcão, A. N.; Pedersen, J. S.; Mortensen, K.; J. Mol. Struct. 1996, 383, 69.

[74] Geissler, E.; Horkay, R.; Hecht, A.-M.; Rochas, C.; Lindner, P.; Borgax, C.; Couarraze, G.; Polymer 1997, 38, 15.

[75] Geissler, E.; Horkay, F.; Hecht, A.-M.; Phys. Rev. Lett. 1993, 71, 645.

[76] Sinha, M.; Mark, J. E.; Jackson, H. E.; Walton, D.; J. Chem. Phys. 2002, 117, 2968.

[77] Urayama, K.; Kawamura, T.; Hirata, Y.; Kohjiya, S.; Polymer 1998, 39, 3827.

[78] Horkay, F.; Hecht, A.-M.; Zrínjyi, M.; Geissler, E.; Polymer Gels and Networks 1996, 4, 451.

[79] Boué, F.; Farnoux, B.; Bastide, J.; Lapp, A.; Herz, J.; Picot, C. L.; Europhys. Lett. 1986, $1,637$.

[80] Pouchelon, A.; George, C.; Menez, P.; Macromol. Symp. 2001, 171, 233.

[81] Kawanura, T.; Urayama, K.; Kohjiya, S.; J. Chem. Phys. 2000, 112, 9105.

[82] Soni, V.; Stein, R.; Macromolecules 1990, 23, 5257. 
[83] Lapp, A.; Leibler, L.; Schosseler, F.; Strazielle, C.; Macromolecules 1989, 22, 2871.

[84] Beshah, K.; Mark, J. E.; Ackerman, J. L.; J. Polym. Sci. B 1986, 24, 1207.

[85] Candau, S.; Peters, A.; Herz, J.; Polymer 1981, 22, 1504.

[86] Herz, J.; Munch, J. P.; Candau, S.; J. Macromol. Sci. - Phys. 1980, B18, 267.

[87] Liberman, M. H.; Abe, Y.; Flory, P. J.; Macromolecules 1972, 5, 550.

[88] Kondo, S.; Igarashi, T.; Polymer 1983, 24, 221.

[89] Adshead, A.; Lindsay, S. M.; Delides, C. G.; King, T. A.; Shepherd, I. W.; Polymer 1979, 20, 329.

[90] Adshead, A.; Lindsay, S. M.; Polymer 1980, 21, 1355.

[91] Lindsay, S. M.; Hartley, A. J.; Shepherd, I. W.; Polymer 1976, 17, 501.

[92] Naoki, M.; Kondo, S.; Polymer 1983, 24, 1139.

[93] Galiatsatos, V.; Mark, J. E.; Macromolecules 1987, 20, 2631.

[94] Bokobza, L.; Nugay, N. J.; J. Appl. Polym. Sci. 2001, 81, 215.

[95] Bokobza, L.; Clement, F. L. M.; Lapersonne, P.; "IR Dichroism and Birefrigence", The Wiley Polymer Networks Group Review, Wiley \& Sons, New York, 1998, vol. 1, chapt. 24.

[96] Lapp, A.; Csiba, T.; Farago, B.; Daoud, M.; J. Phys. II (France) 1992, 2, 1495.

[97] Sotta, P.; Fulber, C.; Demco, D. E.; Blumich, B.; Spiess, H. W.; Macromolecules 1996, 29, 6222.

[98] Brereton, M. G.; Macromolecules 1990, 23, 1119.

[99] McLoughlin, K.; Szeto, C.; Duncan, T. M.; Cohen, C.; Macromolecules 1996, 29, 5475.

[100] McLoughlin, K.; Waldbieser, J. K.; Cohen, C.; Duncan, T. M.; Macromolecules 1997, 30, 1044.

[101] Deloche, B.; Dubault, A.; Herz, J.; Lapp, A.; Europhys. Lett. 1986, 1, 629.

[102] Barth, P.; Hafner, S.; Denner, P.; Macromolecules 1996, 29, 1655.

[103] Simon, G.; Baumann, K.; Gronski, W.; Macromolecules 1992, 24, 3624.

[104] Addad, J. P. C.; Montes, H.; Macromolecules 1997, 30, 3678.

[105] Addad, J. P. C.; Thanh, B. T.; Montes, H.; Macromolecules 1997, 30, 4374.

[106] Sharaf, M. A.; Mark, J. E.; Polymer 1994, 35, 740.

[107] Braun, J. L.; Mark, J. E.; Eichinger, B. E.; Macromolecules 2002, 35, 5273.

[108] Leung, Y. K.; Eichinger, B. E.; J. Chem. Phys. 1984, 80, 3877.

[109] Leung, Y. K.; Eichinger, B. E.; J. Chem. Phys. 1984, 80, 3885.

[110] Lee, K. J.; Eichinger, B. E.; Macromolecules 1989, 22, 1441.

[111] Mark, J. E.; Macromol. Symp. 2001, 171, 1.

[112] Gilra, N.; Cohen, C.; Panagiotopoulos, A. Z.; J. Chem. Phys. 2000, 112, 6910.

[113] Gilra, N.; Panagiotopoulos, A. Z.; Cohen, C.; Macromolecules 2001, 34, 6090.

[114] Malone, S. P.; Vosburgh, C.; Cohen, C.; Polymer 1993, 34, 5149.

[115] Larsen, A. L.; Sommer-Larsen, P.; Hassager, O.; to be published. 
[116] Schimmel, K.-H.; Ph. D. Thesis, Merseburg 1989.

[117] Schimmel, K.-H.; Heinrich, G.; Colloid Polym. Sci. 1991, 269, 1003.

[118] Sharaf, M. A.; Mark, J. E.; Ahmed, E.; Colloid Polym. Sci. 1994, 272, 504.

[119] Mark, J. E.; "Thermoset Elastomers", in "Applied Polymer Science; 21st Century”, Craver, C. D.; Carraher, C. E. Jr.; editors; Am. Chem. Soc., Washington DC 2000, p. 209.

[120] Zhang, L.-X.; Jiang, Z.-T.; Zhao, D.-L.; J. Polym. Sci. B 2001, 40, 105.

[121] Hamurcu, E. E.; Baysal, B. M.; Polymer 1993, 34, 5163.

[122] Queslel, J. P.; Mark, J. E.; J. Polym. Sci. B 1984, 22, 1201.

[123] Mours, M.; Winter, H. H.; Polym. Bull. 1998, 40, 267.

[124] Stepto, R. F. T.; Taylor, D. J. R.; Gottlieb, M.; Polym. Prepr. 1998, 39, 558.

[125] Venkataraman, S. K.; Winter, H. H.; Rheol. Acta 1990, 29, 423.

[126] Tordjeman, P.; Fargette, C.; Mutin, P. H.; J. Rheol. 2001, 45, 995.

[127] Langley, N. R.; J. Non-Crystalline Solids 1991, 131, 894.

[128] Sharaf, M. A.; Mark, J. E.; Polymer Gels and Networks 1993, 33.

[129] Mark, J. E.; Rubber Chemistry and Technology 1999, 72, 465.

[130] Erman, B.; Flory, P. J.; Macromolecules 1982, 15, 806.

[131] James, H. M.; Guth, E. J.; J. Chem. Phys. B 1947, 15, 669.

[132] Langley, N. R.; Macromolecules 1968, 1, 348.

[133] Dossin, L. M.; Graessley, W. W.; Macromolecules 1969, 12, 123.

[134] Fetters, L. J.; Lohse, D. J.; Milner, S. T.; Graessley, W. W.; Macromolecules 1999, 32, 6847.

[135] Orrah, D. J.; Semlyen, J. A.; Ross-Murphy, S. B.; Polymer 1988, 29, 1452.

[136] Aharoni, S. M.; Macromolecules 1986, 19, 426.

[137] Aharoni, S. M.; Macromolecules 1983, 16, 1722.

[138] Rivlin, R. S.; Philos. Trans. Royal Soc., London A 1948, A241, 379.

[139] Termonia, Y.; Macromolecules 1989, 22, 3633.

[140] Venkatraman, S.; J. Appl. Polym. Sci. 1993, 48, 1383.

[141] Edwards, S. F.; Brit. Polymer J. 1977, 140.

[142] de Gennes, P. G.; "Scaling Concepts in Polymer Physics", Cornell University Press, Ithaca, NY 1979.

[143] Doi, M.; Edwards, S. F.; "Theory of Polymer Dynamics", Oxford, Clarendon Press 1986.

[144] Kutter, S.; Terentjev, E. M.; Eur. Phys. J. E 2001, 6, 3, 221.

[145] Kutter, S.; Terentjev, E. M.; Cond-mat/0106371.

[146] Kutter, S.; "Elasticity of polymers with internal topological constraints", Ph. D. thesis, Cambridge University, Aug. 2002.

[147] Higgs, P. G.; Ball, R. C.; Europhys. Lett. 1989, 8, 357.

[148] Edwards, S. F.; Vilgis, T. A.; Polymers 1986, 27, 483. 